\title{
A Neural Network Approach to Writer's Model for Full Likelihood Ratio in Handwriting Analysis
}

\author{
Abiodun Adeyinka Oluwabusayo ${ }^{1,}$, , Adeyemo Adesesan Barnabas ${ }^{2}$ \\ ${ }^{1}$ Department of Computer Science \& Information Technology, Bowen University, Iwo, Nigeria \\ ${ }^{2}$ Department of Computer Science, University of Ibadan, Ibadan, Nigeria
}

Email address:

abiodun.aa12@gmail.com (A. O. Abiodun), sesanadeyemo@gmail.com (A. B. Adeyemo)

${ }^{*}$ Corresponding author

\section{To cite this article:}

Abiodun Adeyinka Oluwabusayo, Adeyemo Adesesan Barnabas. A Neural Network Approach to Writer's Model for Full Likelihood Ratio in Handwriting Analysis. American Journal of Neural Networks and Applications. Vol. 6, No. 2, 2020, pp. 29-35.

doi: 10.11648/j.ajnna.20200602.13

Received: November 4, 2020; Accepted: December 2, 2020; Published: December 16, 2020

\begin{abstract}
Handwriting is an integral part of our life that can predict who we are because the style of writing is unique for every person. Handwriting is also a key element in document examination as it leaves a forensic document examiner with the task of determining who the writer of a particular document is and this is achieved through the likelihood ratio $\left(\mathrm{L}_{\mathrm{R}}\right)$ paradigm. Inability to model an individual's handwriting over time has made estimating a full likelihood ratio for comparative handwriting analysis impossible thereby employing nuisance parameters and subjectivity in computation of $\mathrm{L}_{\mathrm{R}}$ that is not full. This research employed back propagation neural network (BPNN) to model the writing pattern of individuals with input layer as the features of handwriting characters, two hidden layers of three neurons each, activation function sigmoid (s) and an output handwriting. With the help of handwriting model for individual writers, little or no assumptions and no nuisance parameters were employed in achieving full likelihood ratio for comparative handwriting analysis in forensic science. From the research carried out, it can be concluded that modeling an individual's handwriting is a crucial factor in achieving a full likelihood ratio, little/or no inconclusiveness in result reporting and a less degree of disagreements for handwriting identification in a forensic environment.
\end{abstract}

Keywords: $\mathrm{L}_{\mathrm{R}}$, BPNN, Handwriting, Nuisance Parameter, Forensic Handwriting, Document Examination

\section{Introduction}

Handwriting has remained one of the most frequently occurring patterns that we come across in everyday life. It has been established that there are varieties of handwritten documents ranging from forgeries, counterfeiting, identity theft, fraud, suicidal note, contested wills.

A forensic document examiner is saddled with the task of document authenticity. To determine whether a document is genuine, an examiner may attempt to confirm who created the document amongst other things. The likelihood ratio paradigm has been studied as a means for quantifying the strength of evidence for a variety of forensic evidence types in handwriting and other types of forensic evidence such as earmark, speech, footprint, fingerprint, glass fragments and DNA [1-8].
The $L_{R}$ theory takes its stance on odds-form from Bayes ' theorem. Let us presume that there are two opposing ideas. In ' competing, ' it can be said that they are mutually exclusive, but may not necessarily include all possible alternatives. Consider, as an example, a situation where the question is whether a specific writing belongs to a particular person. One proposition is 'The suspect is the author of the document in question'. A counter argument may be' The defendant is not the author of the document in question'. Such two theories are mutually exclusive, but there may be other reasons even if they are far-fetched. For instance, someone might have fabricated the writing with some special skills. The propositions involved should be relevant and the latter case does not seem to be applicable. $L_{R}$ therefore compares the plausibility under two or more propositions and a number of factors are considered when estimating $L_{R}$. It is straightforward for a DNA as compared to other areas of 
forensic evidence because estimation is done for the relative frequency of different DNA profile in relevant populations. For the handwriting evidence, previous literatures reported many factors and the inability of the forensic examiner to model the writing profile of a writer because it is often believed that an individual's writing profile is a latent characteristic that cannot be observed directly, and is not easily modeled because an individual's writing profile cannot have static characteristics and may change over time [9-11] which has resulted to adopt ad-hoc methods to compute likelihood [9, 12, 13] when full likelihood ratio is not achievable.

The purpose of this paper is to illustrate how neural network approach to comparative handwriting analysis can greatly affect the outcome of a forensic investigation and make full likelihood ratio achievable. The novelty of our proposed implementation relies on natural handwriting samples over a period of six months from known individuals to form the database to model the writing profile for each writer. The writer's profile is a very important factor that is considered to accurately estimate a full likelihood ratio.

\section{Overview of a Likelihood Ratio}

The definition of proof analysis is then reflected in the proportion of probabilities. By doing so, the meaning of its value becomes clear, that is, how more (or less) the effects are frequent under the conditions of one proposal than under the conditions of the other proposal.

The probability proportion has been used as a way for measuring the power of confirmation for various legal proof sorts.

$$
\frac{\operatorname{Pr}\left(H_{p} \mid E, I\right)}{\operatorname{Pr}\left(H_{d} \mid E, I\right)}=\frac{g\left(E \mid H_{p}, I\right)}{g\left(E \mid H_{d}, I\right)} X \frac{\operatorname{Pr}\left(H_{p} \mid I\right)}{\operatorname{Pr}\left(H_{d} \mid I\right)}
$$

Formally, posterior belief in favour of $\mathrm{H}_{\mathrm{p}}$ (for example the defendant is guilty) with fair facts are used to make a final decision on the evidence. The forensic examiner provides a summary of the evidence needed for belief based on the evidence and the fact that prior beliefs about $\mathrm{H}_{\mathrm{p}}$ and $\mathrm{H}_{\mathrm{d}}$ have been quantified.

$$
L R \equiv \frac{f\left(a, b \mid H_{p}\right)}{f\left(a, b \mid H_{d}\right)}
$$

$\mathrm{H}_{\mathrm{p}}$ : $\mathrm{a}$ and $\mathrm{b}$ arise from the same source (The writer is the originator of the questioned document)

$\mathrm{H}_{\mathrm{d}}$ : $\mathrm{a}$ and $\mathrm{b}$ arise from different sources (The writer is not the originator of the questioned document)

Likelihood ratio $=$ Posterior ratio $/$ prior ratio

Likelihood ratio according to [14] are increasingly being adopted to convey expert evaluative opinions to courts. In the absence of appropriate databases, many of these likelihood ratios will include verbal rather than numerical estimates of the support offered by the analysis. Computational approaches to the handwriting facet of questioned document
(QD) examination were developed with a view towards providing a scientific basis for handwriting evidence, formalizing human expert-based approaches and validating existing methodology. Extended writing samples such as a paragraph of writing as well as signatures were considered. The task of verification, which is to determine whether two writing samples compared side-by-side, originates from the same person, was the principal problem addressed. A statistical model for writer verification was developed; it allows computing the likelihood ratio based on a variety of different feature types. Strength of proof serves an integral part of this problem. The problem of estimating a $L_{R}$ for handwriting has proven to be a non trival task due to the inability to model the writing pattern of an individual and due to the absence of a large database of handwriting and other factors. [15-18] estimated a $L_{R}$ for handwriting using Bayesian approach but in the presence of nuisance parameter, and their works had no underlying principle and model in which this $L_{R}$ was estimated. [17] based their approach on distribution of measurements from comparing items but their approach was similar to that of marginal $L_{R}$ when full $L_{R}$ was not an option due to the presence of nuisance parameter. In statistics, a nuisance parameter is defined as: "A parameter of a model where there is no scientific intrigue except for whose qualities are normally required (yet when all is said in done are obscure) to make deductions about those parameters which are of such intrigue [25]." [19] described a statistical model for the writer verification task to determine if two documents were written by the same writer, their model followed the scenario proposed by [17] thus suffers from the same weakness [20] presented one conceivable way to deal with gauge a probability proportion in near handwriting analysis was delineated. The oddity of the methodology depends on producing reproduced composing samples from an accumulation of composing samples acquired from a realized source to frame a database for evaluating the dissemination related with the numerator of a $L_{R}$.

In a related work, similar examination of probability proportion based proof appraisal strategies in both evaluative and analytical procedures was carried out using a sample collected from female and male author. While the utilization of probability proportions in the previous circumstance is currently rather entrenched spotlight on the insightful setting still remains rather past contemplations by and by. This paper features that investigative setting still remains rather past contemplations practically speaking; it is also attested that $\mathrm{L}_{\mathrm{R}}$ can be useful for analytical procedures bolstered through various simulations [21].

Assessment of signature handwriting evidence through score-based likelihood ratio based on comparative measurement of relevant dynamic features was carried out by $[22,9]$ presented score-based approaches to calculating forensic probability ratios that have been established as becoming more progressively common in forensic literature. This method is genuinely direct for the score-based $L_{R}$ numerator, which involves creating a list of scores obtained by combining proof objects from the same source. 
Nonetheless, this method creates uncertainty for the generation of denominator databases - in general, how is the best way to produce a list of scores among two items from different source. [23] Asserted that recent analytical developments paired with modern statistical computational tools have led to the proliferation of adhoc techniques for quantifying the probative value of forensic evidence. Many legal and scientific scholars agree that the value of evidence should be reported as likelihood ratio or a Bayes factor. Quantifying the probative value of forensic evidence is subjected to many sources of variability and uncertainty. The work discussed the reasons for the authors' opinion that interval quantifications for the value of evidence should not be used directly in the Bayesian decision making process to determine the support of the evidence for one of the two competing hypotheses. [24] In a related manner, statistical problem and pitfalls identifiable with forensic likelihood ratio were identified.

\section{Methods}

\subsection{Handwriting Modeling}

Figure 1 shows the graphical representation for the handwriting modeling. Original and disguised handwriting were gotten from each writer over a period of six months and a skilled forger was asked to forge these writings. The handwritings were preprocessed using the Otsu method after which they were segmented into different words using the Sobel edge detection algorithm. Each segmented word was then clustered into different characters representing four different groups of upper and lower case alphabets and numbers using the $\mathrm{C}$-means clustering algorithm. Features were extracted via local binary pattern from each clustered characters, while the back-propagation neural network was used to learn the writing pattern for each writer and these writing pattern were then stored in the database.

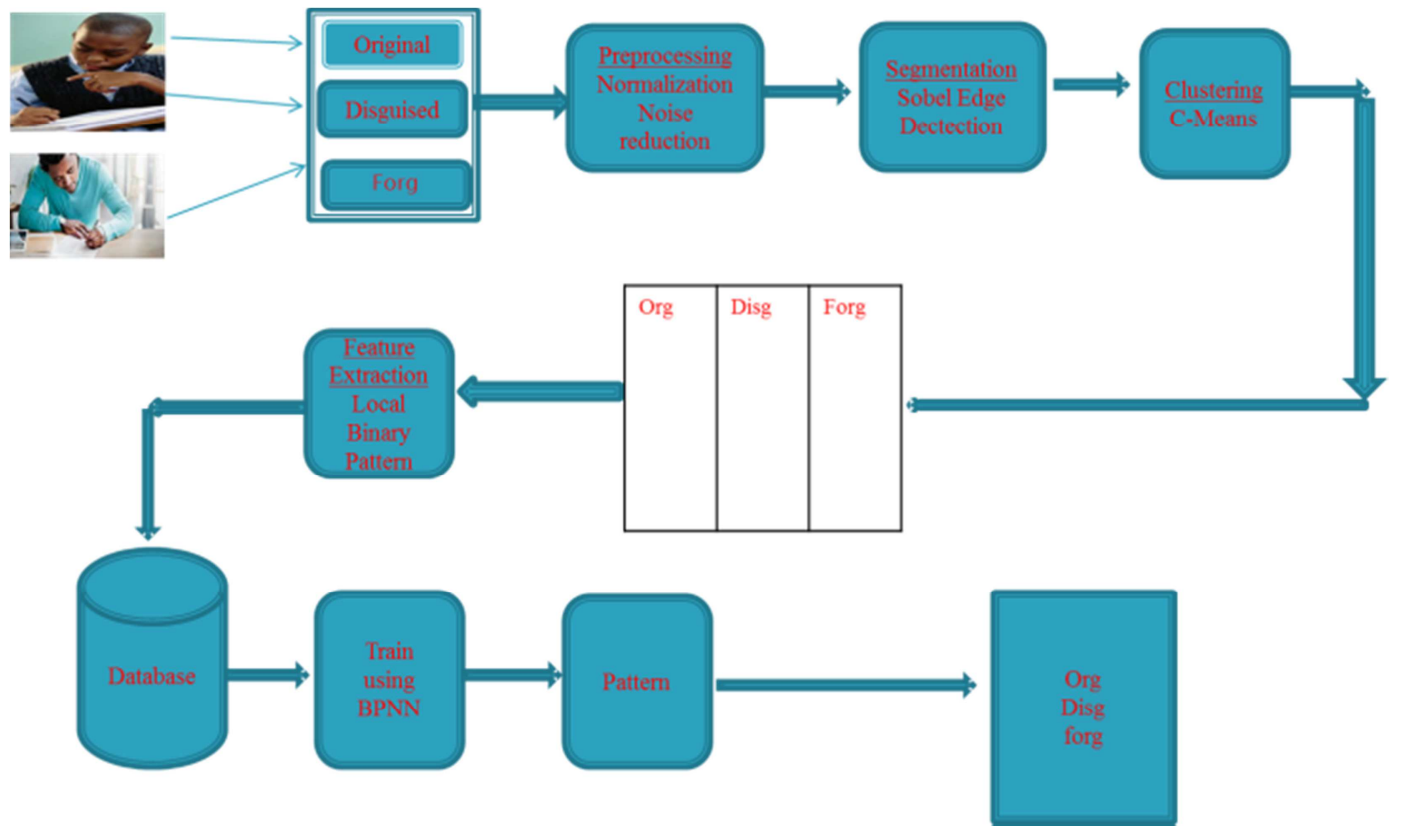

Figure 1. Schematic diagram for the Handwriting character modelling.

\subsection{Back Propagation Neural Network}

Back Propagation Neural Network (BPNN) served the sake of supervised learning of the system. BPNN in the context of this paper was to model the handwriting pattern of each writer over a period of time. The rationale behind the choice of this algorithm is due to the fact that it is a supervised network and a supervised network will have a target, so the BPNN is a network that has a target. We target is set for each character in the handwriting. The target will help to know which handwriting is original and disguised.

BPNN is designed to process information on how the human brain processes information by gathering its knowledge by finding trends and associations in data and learning from experience. Various things come together to form a BPNN, such as hundreds of single units, artificial neurons or processing elements (PE), connected to coefficients (weights), which represent a neural structure and are organized in layers. The strength of neural computation comes from linking neurons in a network. The transition of the neuron functions determines the behavior of the neural networks, the laws of learning and the structure itself. The neural network is a parametrized system because the weights are variable parameters. The weighted number of the inputs is the activation of the neuron. The activation signal is passed through the transfer feature to produce a single neuron output. Transfer function can add non-linearity to the network. During practice, inter-unit connections are optimized until the forecast error is reduced and the network achieves the required level of accuracy. In terms of model configuration, artificial neural networks do not require knowledge of the data source, but require large training sets because they often include several weights that need to be calculated. In addition, that each individual's writing pattern 
was modeled using BPNN with the algorithm below, as BPNN can combine and incorporate both literature-based and experimental data to solve problems.

\subsubsection{Back Propagation Neural Network Implementation}

Each character variable has a weight $W_{i}$ which shows its contribution in the training process. The feature information extracted from student handwriting through LBP was entered in NN through input layer and the participation of each class of character variables is calculated by the hidden layer of the network using:

$$
n e t_{j}=\sum_{i=1}^{n+1} w_{i j}^{(1)} \hat{o}_{i}
$$

$n e t_{j}$ is $i t h$ type of factor, $n$ is the number of factors in $n e t_{i}$, $\hat{o}_{i}$ weight-recognition factor with weight $w_{i j}^{(1)}$.

This study used a network concealed with $\mathrm{n}$ input points, $\mathrm{k}_{1}$ and $\mathrm{k}_{2}$ hidden, and $m$ output units. The weight from input points $i$ and two hidden unit $j$ is $w_{i j}^{(1)}$ and $w_{i j}^{(2)}$. Weight from second hidden unit $i$ and output unit $j$ is $w_{i j}^{(3)}$. Weight of additional edge for each unit is bias $-\theta$, where input unit and output vector from the hidden layer are expanded with a 1 component as seen in Figure 2. Weight from the constant 1 and two hidden unit $\mathrm{j}$ is $w_{n+1, k 1}^{(1)}$ and $w_{k 1+1, k 2}^{(2)}$. by $w_{k 2+1, m}^{(3)}$ is the weight from constant 1 and output unit $j$. There are $(n+$ 1) $\times k$ weights between input sites and first hidden units, $(k 1$ $+1) \times k 2$ weights between first input sites and second hidden units and $(k 2+1) \times m$ between hidden and output units. Let $\overline{w_{1}}$ denote the $(n+1) \times k$ matrix with component $w_{i j}^{(1)}$ at the $i$ th row and the $j$-th column and $\overline{w_{2}}$ denote the $(k 1+1) \times k 2$ matrix with component $w_{i j}^{(2)}$ at the $i$-th row and the $j$-th column. Similarly let $\overline{w_{3}}$ denote the $(k 2+1) \times m$ matrix with components $w_{i j}^{(3)}$. The study used an overlined notation to emphasize that the last row of both matrices corresponds to the biases of the computing units. The matrix of weights without this last row will be needed in the backpropagation step. The n-dimensional input vector $o=\left(o_{1}, \ldots, o_{n}\right)$ is extended, transforming it to $\hat{o}=\left(o_{1}, \ldots, o_{n}, 1\right)$. The excitation $n e t_{j}$ of the $j$-th hidden unit is given by

$$
n e t_{j}=\sum_{i=1}^{n+1} w_{i j}^{(1)} \hat{o}_{i}
$$

The activation function is a sigmoid $(s)$, the output $o_{j}^{(1)}$ is thus

$$
o_{j}^{(1)}=s\left(\sum_{i=1}^{n+1} w_{i j}^{(1)} \hat{o}_{i}\right)
$$

The excitation of all units in the two hidden layers can be computed with the vector-framework $\hat{o} \bar{w}_{1}$ and $\hat{o} \bar{w}_{2}$. increase. The vectors $o^{(1)}$ and $o^{(2)}$ whose segments are the output of the two hidden units is given by

$$
\begin{aligned}
& o^{(1)}=s\left(\hat{o} \bar{w}_{1}\right) \\
& o^{(2)}=s\left(\hat{o} \bar{w}_{2}\right)
\end{aligned}
$$

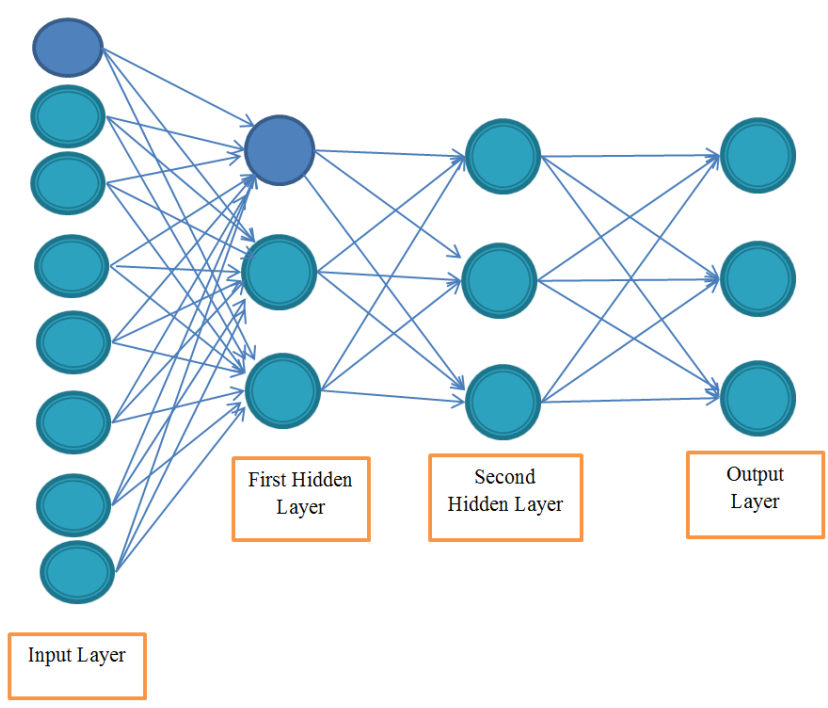

Figure 2. Neural Network for the Recognition of Handwriting Diagram.

Using the convention of applying the sigmoid to each component of the argument vector. The excitation of the units in the output layer is computed using the extended vector $\hat{o}^{(1)}=\left(o_{1}^{(1)}, \ldots, o_{k}^{(1)}, 1\right)$. The output of the network is the $\mathrm{m}$ dimensional vector

Result of the output layer represents an overall output of recognition by the NN component which is given by:

$$
o^{(3)}=s\left(\hat{o}^{(1)} \bar{w}_{3}\right)
$$

\subsubsection{Algorithm}

After randomly selecting the weights of the network, the backpropagation algorithm is used to measure the necessary corrections. The algorithm can be broken down in the following four steps:

Feed-forward computation

Backpropagation to the output layer

Backpropagation to the hidden layer

Weight updates

The algorithm is stopped when the value of the error function has become sufficiently small.

Step 1: Feed-Forward Computation

The vectors $o^{(1)}, o^{(2)}$ and $o^{(3)}$ are computed and stored, evaluated derivative also stored.

Step 2: Backpropagation to the output layer

This research looked for the first partial derivatives $\partial E / \partial w_{i j}^{(3)}$. The backpropagated error $\delta_{j}^{(3)}$ is defined by

$$
\delta_{j}^{(3)}=o_{j}^{(3)}\left(1-o_{j}^{(3)}\right)\left(o_{j}^{(3)}-t_{j}\right)
$$

where $o_{j}^{(3)}$ is the output and $t_{j}$ is the target and the partial derivative this research looked for is 


$$
\partial E / \partial w_{i j}^{(3)}=\left[o_{j}^{(3)}\left(1-o_{j}^{(3)}\right)\left(o_{j}^{(3)}-t_{j}\right)\right] o_{i}^{(1)}=\delta_{j}^{(3)} o_{i}^{(1)}
$$

Step 3: Backpropagation to the hidden layer

Partial derivatives $\partial E / \partial w_{i j}^{(1)}$ is calculated by connecting

each unit $\mathrm{j}$ in the two hidden layer to the output layer having an edge of weight $w_{j q}^{(2)}$, where $q=1, \ldots, m$. This is the backpropagated mistake

$$
\delta_{j}^{(1)}=o_{j}^{(1)}\left(1-o_{j}^{(1)}\right) \sum_{q=1}^{m} w_{j q}^{(2)} \delta_{j}^{(2)}
$$

The partial derivative is therefore

$$
\partial E / \partial w_{i j}^{(1)}=\delta_{j}^{(1)} o_{i}
$$

The back-propagated error can be resolved similarly for any number of hidden layers and the expression for the partial derivatives of $E$ holds the equivalent analytical form

Step 4: Weight Updates

In the wake of registering every single partial derivatives, the net weights are changed in the negative gradient direction. The learning constant decides the length of the correlation step. Corrections to the weights are made by

$$
\Delta w_{i j}^{(2)}=-\gamma o_{j}^{(1)} \delta_{j}^{(2)}, \text { for } i=1, \ldots, k+1 ; j=1, \ldots, m,
$$

And

$$
\Delta w_{i j}^{(2)}=-\gamma o_{i} \delta_{j}^{(1)}, \text { for } i=1, \ldots, n+1 ; j=1, \ldots, k,
$$

This study used the knowledge that $o_{n+1}=o_{k+1}^{(1)}=$ 1. Weight corrections is important only after the backpropagated error has been computed for all units in the network otherwise, the corrections will be entangled with the backpropagation of the error and the calculated corrections will no longer conform to the negative gradient line.

\section{Results}

Due to the complexity of modeling the handwriting of a writer and the absence of industrial size databases from which different handwriting can be described [17, 9] estimated a marginal $L_{R}$ when the full $L_{R}$ was not possible also in the presence of some parameters considered to be nuisance. This research work was able to model the handwriting for individual in the presence of large-scale database using the back-propagation neural network (BPNN). Each scanned, segmented and clustered characters and alphabets which were collated over a period of six months were trained to learn the pattern for each writer. The developed writing model for each writer is one of the criteria to eliminate the presence of nuisance parameters when estimating a full $\mathrm{L}_{\mathrm{R}}$.

Table 1 shows results of an estimated likelihood ratio for a suspect against every other suspect in the pool of database.

Base on decision law i.e.

If $L_{R}$ value greater than $1 \mathrm{H}_{\mathrm{p}}$ is true

If $\mathrm{L}_{R}$ value less than $1 \mathrm{H}_{\mathrm{p}}$ is false. An inconclusive state is declared if and only if $L_{R}$ value $=1$

With the BPNN algorithm to model handwriting pattern for each writer and $L_{R}$ estimation described in [10] this paper was able to deal with the problem of inconclusiveness as there was no inconclusive results reported in the investigation, determine who the writer of a questioned document is as well as eliminate the inclusion of parameters considered nuisance in investigation because each writer's handwriting profile could be ascertained due to the BPNN training of the Handwriting categories. It has also helped in lowering the rate of disagreement $\left(L_{R} 7\right.$ and 10) in the investigation process as compared to existing methods. A more elaborate and collated result table is presented in Table 2.

Table 1. A comparison of the approximate $L_{R}$ of the perpetrator to every other suspect in the pool of the applicable database.

\begin{tabular}{llll}
\hline & EL & CiL & CiU \\
\hline $\mathrm{L}_{\mathrm{R}} 1$ & 1.5387 & -8.86922 & 11.94662 \\
$\mathrm{~L}_{\mathrm{R}} 2$ & 9.4649 & -0.94302 & 19.87282 \\
$\mathrm{~L}_{\mathrm{R}} 3$ & 1.6004 & -8.80752 & 12.00832 \\
$\mathrm{~L}_{\mathrm{R}} 4$ & 1.9914 & -9.31652 & 11.49932 \\
$\mathrm{~L}_{\mathrm{R}} 5$ & 1.6281 & -8.77982 & 12.03602 \\
$\mathrm{~L}_{\mathrm{R}} 6$ & 1.533 .4 & -9.40792 & 11.40792 \\
$\mathrm{~L}_{\mathrm{R}} 7$ & 0.8807 & -9.52722 & 11.28862 \\
$\mathrm{~L}_{\mathrm{R}} 8$ & 19.2808 & 8.872876 & 29.68872 \\
$\mathrm{~L}_{\mathrm{R}} 9$ & 1.3336 & -9.07432 & 11.74152 \\
$\mathrm{~L}_{\mathrm{R}} 10$ & 0.8907 & -9.51722 & 11.29862 \\
$\mathrm{~L}_{\mathrm{R}} 11$ & 1.2135 & -9.19442 & 11.62142 \\
$\mathrm{~L}_{\mathrm{R}} 12$ & 1.9341 & -9.47382 & 11.34202 \\
$\mathrm{~L}_{\mathrm{R}} 13$ & 2.9482 & -9.45972 & 11.35612 \\
$\mathrm{~L}_{\mathrm{R}} 14$ & 1.9188 & -8.48912 & 12.32672 \\
$\mathrm{~L}_{\mathrm{R}} 15$ & 1.3209 & -9.08702 & 11.72882 \\
- & - & - & - \\
\hline- & - & - & - \\
$\mathrm{L}_{\mathrm{R}} 229$ & 0.0019 & -10.406 & 10.40982 \\
\hline
\end{tabular}

Statistically the estimation of the interval is considered to be robust over the estimation point, therefore we do consider the estimation of the interval and take into account the decision condition, and we conclude that both the down and top intervals must be of the same sign; that is, either both positive or both negative. Thus there must be agreement in sign otherwise there is disagreement. Table 1 shows the results of our Estimated Likelihood Ratio (ELR) for a writer against any other author in a collection of appropriate databases where the upper confidence interval (CiU) and the lower confidence interval (CiL) are indicated. 
Table 2. A table showing the estimated $L_{R}$ for each writer in the pool of the applicable database.

\begin{tabular}{llllll}
\hline & KDE & & & \\
\hline Case & Support Hp (LR>1.00) & Against Hp (LR < 100) & LCI (95\%) Support Hp & UCI (95\%) Support Hp & Disagreement \\
\hline 1 & 96.67 & 3.33 & 92.38 & 98.82 & 3.33 \\
2 & 94.12 & 5.88 & 90.38 & 97.47 & 5.88 \\
3 & 87.50 & 12.5 & 82.97 & 94.67 & 12.5 \\
4 & 91.67 & 8.33 & 87.42 & 95.27 & 8.33 \\
5 & 96.43 & 3.57 & 94.86 & 98.27 & 3.57 \\
6 & 85.71 & 14.29 & 81.71 & 90.94 & 14.29 \\
7 & 87.50 & 12.5 & 83.27 & 93.29 & 12.5 \\
8 & 90.91 & 9.09 & 88.16 & 94.75 & 2.38 \\
9 & 97.62 & 2.38 & 95.29 & 99.86 & 7.14 \\
10 & 92.86 & 7.14 & 89.39 & 95.39 & \\
11 & 95.72 & 4.28 & 91.19 & 98.24 & 1.11 \\
12 & 88.89 & 11.11 & 85.87 & 95.28 & \\
\hline
\end{tabular}

\section{Conclusion}

Full $L_{R}$ void of nuisance parameters is needed for most forensic investigators. Several factors have to be put in place to estimate a full $\mathrm{L}_{\mathrm{R}}$ for forensic handwriting investigations. This research shows that modeling handwriting pattern for a writer is an important factor in achieving a full $L_{R}$ which will eliminate the use of nuisance parameters in the computation process, lower the disagreement rate with respect to the Hypothesis in support of the prosecutor and also produce no inconclusive result after estimation of $\mathrm{L}_{\mathrm{R}}$.

\section{References}

[1] C. Champod, I. W. Evett, B. Kuchler, Earmarks as evidence: a critical review, J. Forensic Sci. 46 (2001) 1275-1284.

[2] C. Neumann, C. Champod, R. Puch-Solis, N. Egli, A. Anthonioz, A. Bromage-Griffiths, Computation of likelihood ratios in fingerprint identification for configurations of any number of minutiae, J. Forensic Sci. 52 (2007) 54-64.

[3] E. Enzinger (2016). Likelihood ratio calculation in acousticphonetic forensic voice comparison: Comparison of three statistical modelling approaches. Proceedings of the Interspeech 2016, 535-539.

[4] Ruthmara Corzo, Tricia Hoffman, Peter Weis, Javier FrancoPedroso, Daniel Ramos, Jose Almirall (2018)'The use of LAICP-MS databases to calculate likelihood ratios for the forensic analysis of glass evidence', qq Talanta, Volume 186, Pages 655-661, ISSN 0039-9140.

[5] Cedric Neumann, Christopher P. Saunders, Commentary on: Alberink I, de Jongh A, Rodriguez C. (2015) Fingermark evidence evaluation based on automated fingerprint identification system matching scores: the effect of different types of conditioning on likelihood ratios. J Forensic Sci 2014; 59 (1): 70-81., Journal of Forensic Sciences, 10.1111/1556-4029.12634, 60, 1, (252-256).

[6] R. Marquis, S. Bozza, M. Schmittbuhl, F. Taroni, Handwriting evidence evaluation based on the shape of characters: application of multivariate likelihood ratios, J. Forensic Sci. 56 (2011) S238-S242.

[7] Hepler AB, Saunders CP, Davis LJ, Buscaglia J (2012) Scorebased likelihood ratios for handwriting evidence. Forensic Sci
Int 219: $129-40$.

[8] Abiodun A. O, Adeyemo A. B (2019) An Exhaustive Mapping Model for Modified Likelihood Ratio for Handwriting Recognition in Forensic Science. J Forensic Sci Criminol 7 (3): 301 ISSN: 2348-9804.

[9] Alberink I, de Jongh A, Rodriguez C (2013) Fingermark evidence evaluation based on automated fingerprint identification system matching scores: the effect of different types of conditioning on likelihood ratios. J Forensic Sci 59: 70-81.

[10] Cook R, Evett IW, Jackson G, Jones PJ, Lambert JA (1998) A method for case assessment and interpretation. Sci Just 38: $151-6$.

[11] Royall RM (1997) Statistical Evidence: A Likelihood Paradigm, Chapman \& Hall, London.

[12] Martire K. A., Kemp R. I., Sayle M., \& Newell B. R. (2014). On the interpretation of likelihood ratios in forensic science evidence: Presentation formats and the weak evidence effect. Forensic Science International, 240, 61-68. https://doi.org/10.1016/j.forsciint.2014.04.005.

[13] Marquis R., Bozza S., Schmittbuhl M., Taroni F., (2011) Handwriting evidence evaluation based On the shape of characters: Application of multivariate likelihood ratios, Journal of Forensic Science 56, S238-S242.

[14] Srihari, S. N., Cha, S., Arora, H., and Lee, S. (2002). Individuality of Handwriting. Journa of Forensic Science, 47 (4), 1-17.

[15] Aitken G. G. and Taroni F. (2004). Statistics and the Evaluation of Evidence for Forensic Scientists: 2nd edition. John Wiley and Sons.

[16] Bozza S., Taroni F., Marquis M., Schmittbuhi, (2008) 'Probabilistic evaluation of handwriting evidence: likelihood ratio for authorship' J. R. Stat. Soc. Ser. C: Appl. Stat. 57329 341.

[17] Srihari S. N, Beals M. J, Bandi K. Shah V. (2005) A statistical model for writer verification, In: Proceedings of the Eighth International Conference on Document Analysis and Recognition, IEEE Computer Society, pp. 1105-1109.

[18] Davis, L. J., Saunders, C. P., Hepler, A., and Buscaglia, J. (2012). Using subsampling to estimate the strength of handwriting evidence via score-based likelihood ratios. Forensic Science International, 216 (1-3), 146-157. https://doi.org/10.1016/j.forsciint.2011.09.013. 
[19] Taroni, F., Marquis, R., Schmittbuhl, M., Biedermann, A., Thiéry, A., and Bozza, S. (2012). The use of the likelihood ratio for evaluative and investigative purposes in comparative forensic handwriting examination. Forensic Science International, $\quad 214 \quad(1-3), \quad 189-194$. https://doi.org/10.1016/j.forsciint.2011.08.007.

[20] Chen X., Champod, C., Yang, X., Shi, S., Luo, Y., Wang, N. (2018). Assessment of signature handwriting evidence via score-based likelihood ratio based on comparative measurement of relevant dynamic features. Forensic Science International, 282 ,

101-110.
[21] Ommen D. M., Saunders, C. P., \& Neumann, C. (2016). Science and Justice An argument against presenting interval quantifications as a surrogate for the value of evidence. Science \& Justice, 56 (5), 383-387. https://doi.org/10.1016/j.scijus.2016.07.001.

[22] Dawid A. P. (2017). Science and Justice Forensic likelihood ratio: Statistical problems and pitfalls. Science \& Justice, 57 (1), 73-75. https://doi.org/10.1016/j.scijus.2016.09.002.

[23] B. S Everitt. The Cambridge Dictionary of Statistics. $3^{\text {rd }}$ Ed. Cambridge University Press. Cambridge, UK 2006. 